\title{
El reloj, la hora y la economía del tiempo en La Celestina
}

\author{
Enrique Fernández Rivera \\ University of Manitoba
}

Ya Lida de Malkiel señaló que uno de los rasgos de modernidad de La Celestina es lo que ella llama "su aguda conciencia de tiempo» y su "profusión de tiempos concretos». ${ }^{1}$ Efectivamente, es notable en la obra la abundancia de referencias al tiempo, a cuándo se realizan las acciones y a su duración. Esta precisión no era común en la literatura anterior, la cual tendía a presentar el aspecto temporal de la acción de manera más vaga. La aguda conciencia temporal de La Celestina se trasluce también en cómo los personajes se muestran en muchas ocasiones preocupados por el tiempo, sea en frases sentenciosas sobre el paso acelerado de los años o en referencias a la puntualidad, a la hora que es, etc. Esta notable presencia del tiempo en la obra se ha estudiado desde diferentes ángulos. Una parte de estos estudios se ha concentrado en lo que podríamos llamar el tiempo interno de la obra, es decir, las jornadas que cubre la acción, el tiempo implícito y sus faltas de consistencia, así como las implicaciones de cara al género de La Celestina. Otros estudios han prestado atención a cómo los personajes experimentan el tiempo, a sus quejas de lo efímero de la vida o a su impaciencia porque el tiempo no pase al ritmo que a ellos les gustaría, especialmente antes de las anheladas citas amorosas. ${ }^{2}$ Finalmente, un grupo de estudios ha examinado la percepción del tiempo en la obra y su

1.- María Rosa Lida de Malkiel, La originalidad artística de "La Celestina», Buenos Aires, Eudeba, 1962, pp. 169 y 171.

2.- Además de los comentarios que Lida de Malkiel hace al respecto en la obra citada (véase en particular el capítulo siete, titulado precisamente "El tiempo», pp. 149-168), otros estudios que tratan el tiempo desde este ángulo son Manuel J. Asensio, «El tiempo en La Celestina», en Hispanic Review 20.1 (1952), pp. 28-43, y Stephen Gilman, "A Propos of 'El tiempo en La Celestina' by Manuel J. Asensio», en Hispanic Review 21.1 (1953), pp. 42-45. El reciente artículo de Florencio Sevilla Arroyo, "Amor, magia y tiempo en La Celestina», en Celestinesca 33 (2009), pp. 173-214, contiene una revisión bibliográfica puesta al día de las visiones del tiempo en la obra. 
correspondencia con la forma de vida de los nuevos centros urbanos y la incipiente burguesía que en ellos habitaba. La siguiente cita de Maravall resume bien este punto de vista:
[D]e acuerdo con esta caracterización urbana del mundo de La Celestina, observemos que sus personajes viven su tiempo medido y regulado por el reloj. Quiero decir que el reloj es el instrumento de que se sirven para medir y ordenar su tiempo. Son numerosas las referencias al reloj que se encuentran en el texto de la Tragicomedia: para co- mer, para estar en la cama, para acudir a una cita amoro- sa, para medir una espera, el reloj aparece una y otra vez con su movimiento mecánico, uniforme, calculado. [...] Responde a la concepción mecánica, calculable, mensu- rable, del tiempo y, en general, del mundo, propia de los burgueses que habitan la gran ciudad. De aquí que, en éstas, se convierta en un elemento común de la arquitec- tura pública, de la misma manera que se generaliza en la vida privada de sus moradores. De esta última forma, lo descubrimos rigiendo cronológicamente la existencia de nuestros personajes. ${ }^{3}$

Esta larga cita viene a cuento porque mi intención es estudiar en detalle cómo funciona y qué implica en La Celestina esta omnipresencia del reloj como tecnología que permite saber la hora del día. Trataré pues del tiempo en su sentido más concreto y cotidiano de saber qué hora es, de cuánto tiempo falta para que sea una hora determinada y otro tipo de operaciones relacionadas con el cálculo y uso del tiempo que, por ser tan similares a como hoy seguimos administrando el tiempo, tienden a pasar desapercibidas. Quiero por tanto prestar atención a cómo la obra es afectada por la incipiente tecnología del reloj mecánico y su forma precisa de medir el tiempo. Como veremos, el reloj mecánico y la nueva sensibilidad hacia la hora están claramente presentes en la obra. Desde el punto de vista de la estructura de la obra, sirven para agilizar el desarrollo de la trama. Al mismo tiempo, refuerzan al nivel concreto de la acción una serie temas fundamentales de La Celestina, como la fugacidad del tiempo y la necesidad de saber usarlo.

En La Celestina, la descripción de la medición y economía del tiempo, es decir, del día a día de cómo organizar la vida, es un retrato fidedigno de la vida en las ciudades de la época. En este escenario urbano el reloj mecánico -llamado así para distinguirlo del reloj solar o astronómico y de los relojes de agua y arena- era un instrumento importante por la precisión y la regularidad que ofrecía para organizar y coordinar las ac- 
tividades cada vez más complejas de la vida diaria. La obra describe un momento histórico muy concreto en que la necesidad de saber la hora precisa se estaba imponiendo, ofreciéndonos así una de las primeras instancias de la compulsión por saber la hora exacta en todo momento que hoy compartimos. Sin embargo, frente a esa incipiente necesidad de estar al tanto de la hora, la tecnología horaria disponible era imprecisa y poco práctica. A finales del xv, en las ciudades y pueblos grandes los relojes mecánicos llevaban más de un siglo instalados en campanarios y edificios públicos. ${ }^{4}$ Por testimonios indirectos tenemos conocimiento de cómo los centros urbanos fueron reemplazando con relojes mecánicos los relojes de sol que solía haber en las torres de iglesias y otros edificios de las plazas. Poco a poco, el sistema de veinticuatro horas sustituyó al de las horas canónicas y la hora pasó a hacerse completamente laica, lo que se ha llamado la transición del tiempo sagrado al «tiempo de los mercaderes». ${ }^{5}$

A finales del siglo xv, cuando se escribió La Celestina, era inevitable que una ciudad del tamaño de la que se sobreentiende en la obra tuviera uno de estos relojes públicos para servir como marcador del ritmo colectivo de una vida urbana que ya mostraba rasgos de modernidad. En la obra hay un reloj implícito situado en un lugar elevado, sea una iglesia o una torre de otro edificio, que da las horas con campanadas que se oyen por toda la ciudad. Estas señales horarias eran muy importantes porque los relojes en las casas eran muy escasos o casi inexistentes - está claro que Calisto no tenía ninguno en casa-y los relojes portátiles para llevar encima aún no se habían inventado. La única forma de saber la hora era pues escuchar las campadas de un reloj que sólo se podía ver desde la plaza y sus inmediaciones pero cuyas campanadas horarias se podían oír con claridad por toda la ciudad. ${ }^{6}$

4.- Algunos autores señalan la década de 1450 como las fecha en que este nuevo tipo de vida regulado por el reloj se convierte en la norma, cfr. Gerhard Dohrn-van Rossum, History of the Hour: Clocks and Modern Temporal Orders, Chicago, University of Chicago Press, 1996, p. 273. Existe una copiosa bibliografía sobre la historia del reloj. Obras clásicas y en cuyos datos me baso son Carlo M. Cipolla, Clocks and Culture, 1300-1700, London, Collins, 1967, y Gerhard Dohrn-van Rossum, cit. Para España es muy útil la colección de seis volúmenes editada por Luis Montañés Fontenla, Biblioteca literaria del relojero, 6 vols., Madrid, R. Carbonell Blasco, 1953-58.

5.- Jacques Le Goff, "Merchant's Time and Church's Time in the Middle Ages», en Time, Work and Culture in the Middle Ages, ed. Jacques Le Goff, Chicago, University of Chicago Press, 1980, pp. 29-42.

6.- Durante el siglo XVI se fueron introduciendo lentamente los relojes de mesa y de pared en las casas particulares, aunque en un principio sólo los reyes, la nobleza y los más adinerados los poseían, véase para el caso de España José A. García-Diego, Los relojes y autómatas de Juanelo Turriano, Madrid, Albatros, 1982. Relojes portátiles existieron ya desde mediados del XVI, aunque eran escasos y costosos hasta varios siglos después El uso del reloj de pulsera se introdujo sólo a principios del siglo xx conectado con su uso durante la Primera Guerra Mundial por los soldados en las trincheras. 
Las campanadas horarias de este reloj público son el telón de fondo de La Celestina, sea por su presencia audible o por el anhelo con el que los personajes las esperan. En varias ocasiones el texto refleja cómo los personajes escuchan las campanadas de este reloj. La expresión que se utiliza para esta acción del reloj es la misma que usamos hoy, «dar la hora». Por ejemplo, Calisto, en su cita nocturna, al acercarse a casa de Melibea, dice a Pármeno: «Las doce da ya» (241). ${ }^{7}$ El sujeto del verbo «dar» es el reloj que está implícito en esta frase y que aparece de manera explicita en otros pasajes, como cuando Calisto dice a Melibea tras la noche de amor en el huerto: "Ya quiere amanecer. ¿Qué es esto? No parece que ha una hora que estamos aquí y da el reloj las tres» (275, énfasis añadido).

La presencia del reloj mecánico en La Celestina no se limita a estos y otros pasajes similares en que se mencionan las campanadas horarias. Cuando el impaciente Calisto se queja de la lentitud con que pasa el tiempo que falta para su cita con Melibea, alude al reloj mecánico distinguiéndolo en la forma de llamarlo claramente de los relojes solares, de agua o de arena aún comunes en la época: "¿Qué me aprovecha a mí que dé doce horas el reloj de hierro si no las ha dado el del cielo?» (282, énfasis añadido). ${ }^{8} \mathrm{El}$ «reloj de hierro» es el nombre que usa para el reloj mecánico. La referencia al hierro es interesante pues refleja el estado de la tecnología relojera en la época. Los relojes mecánicos de madera habían sido ya arrinconados siglos antes por el reloj de hierro, pero el reloj de bronce o latón, que acabaría imponiéndose por permitir una tecnología más precisa y portátil, aún no era común. Igualmente, este comentario de Calisto resalta otra peculiaridad del estado de la tecnología horaria del momento: la imprecisión de los relojes por su propensión a adelantarse o atrasarse de manera considerable con respecto al tiempo real o tiempo astronómico fielmente indicado por el sol y las estrellas ${ }^{9}$

7.- Énfasis añadido. Esta cita y todas las siguientes son por número de página a Fernando de Rojas y "Antiguo Autor», La Celestina, tragicomedia de Calisto y Melibea, ed. Guillermo Serés, Francisco J. Lobera, Paloma Díaz-Mas, Carlos Mota, Iñigo Ruiz Arzálluz y Francisco Rico, Barcelona, Crítica, 2000.

8.- En otra ocasión se menciona un reloj mecánico que da campanadas, aunque la referencia es sólo una comparación de Calisto con un reloj que sirve para expresar su aturdimiento: «Ya escurre eslabones el perdido; ya se desconciertan sus badajadas. Nunca da menos de doce; siempre está hecho reloj de medio día» (148).

9.- La imprecisión de los relojes — que no se solucionaría hasta casi un siglo después cuando el holandés Huygens introdujo el péndulo- era queja muy común en la época. Para la imprecisión de los relojes antes de las innovaciones introducidas por Huygens véase $\mathrm{H}$. von Bertele, "Precision Timekeeping in the Pre-Huygens Era», en The Horological Journal 95 (1953), pp. 794-816. Esta imprecisión se refleja en la obra del vallisoletano Arce de Otálora Los coloquios de Palatino y Pinciano, escrita hacia 1550, en la que, tras mencionar lo poco fiables que son los relojes públicos de Salamanca y Simancas, se concluye: «Muy más cierta es [la astrología] para quien la entiende que el mejor reloj de Salamanca, porque el del cielo nunca se desconcierta ni puede desconcertar, y los de la tierra casi nunca están concertados, si no es el de Bolonia, que dicen que tiene por badajo un hombre que no hace más de regirse de día 
No es de extrañar que, dada la imprecisión de los relojes de la época, la habilidad de leer la hora por el sol y los astros aún esté presente en $\mathrm{La}$ Celestina. La obra contiene claros restos de lo que podemos llamar «bilingüismo horario" de sus personajes, es decir, su capacidad de poder comprobar la hora por el sol y las estrellas. En varias escenas los personajes hacen gala de esta habilidad. Cuando Pármeno se despierta tras la noche de amor con Areúsa, por la posición alta del sol se da cuenta de que es mucho más tarde de lo que creía (187-88). Igualmente, la constelación de las Pléyades - llamadas las Cabrillas en la obra—, la Estrella Polar -Norte- y el planeta Venus - lucero del alba - son mencionados por Celestina para describir a las jóvenes enamoradas impacientes por la hora a la que ha de ir o venir su amante durante la noche: "[M]aldicen los gallos porque anuncian el día y el reloj porque da tan apriesa. Requieren las Cabrillas y el Norte, haciéndose estrelleras; ya cuando ven salir el lucero del alba, quiéreseles salir el alma. Su claridad les escuresce el corazón» (103). ${ }^{10}$ Un curioso resto de esta capacidad de saber la hora por el cielo se refleja en varias instancias de la expresión "ver la hora», que claramente se refiere a ver la posición del sol. Así ocurre cuando Calisto, al día siguiente de su visita nocturna a Melibea, se despierta y ordena a Sosia: «[A]bre esa ventana, verás qué hora es» (264, énfasis añadido).

A pesar de la supervivencia de la antigua práctica de saber la hora por el sol o las estrellas, en La Celestina predomina un uso sistemático de horas exactas para arreglar las citas y coordinar la vida que sólo es posible merced al empleo generalizado del reloj mecánico. No sólo los amantes Calisto y Melibea usan la hora precisa de las doce de la noche para encontrarse sino que también los demás personajes emplean el reloj mecánico para establecer encuentros a horas determinadas, y no vagamente "al atardecer», "a media tarde», o "al caer la noche», como era el caso en obras anteriores. Así, Sempronio se queja del retraso de Celestina, a la que espera "desde que dio la una» (139); la comida de Pármeno y Sempronio en casa de Celestina con Areúsa y Elicia se establece para las doce del día, aunque luego ellos llegan tres horas tarde (188, 203); Sosia lleva sus caballos a beber antes de las diez (304); el enamorado Calisto duerme

por el sol y de noche por la luna y norte, y dar con un martillo de hierro en una campana las horas a su tiempo" (Coloquios de Palatino y Pinciano, ed. José Luis Ocasar Ariza, 2 vols., Madrid, Turner, 1995, vol. II, p. 968).

10.- Curiosamente Celestina se muestra en este comentario algo desfasada ya que el reloj de la ciudad en la que ocurre la acción de la obra sigue dando sus campanadas horarias durante la noche, como se deja claro, por ejemplo, en las palabras antes citadas de Calisto de que el reloj ha dado las tres y el amanecer está cercano. Sobre los varios métodos de ver la hora por la astros y su uso antes del reloj mecánico en occidente y en otras culturas, véase Nina Gockerell, "Tellling Time without a Clock», en The Clockwork Universe: German Clocks and Automata 1550-1560, eds. Klaus Maurice y Otto Mayr, New York, Neal Watson Academic Publishing, 1980, pp. 131-43. 
hasta las cuatro de la tarde (283), y un largo etcétera de eventos a horas concretas que se nos da en la obra. ${ }^{11}$

La precisión horaria que caracteriza la obra no alcanza, sin embargo, a incluir fracciones más pequeñas de la hora, como medias horas y cuartos de hora. Esta ausencia responde a la realidad de los relojes de la época, que tenían solamente una aguja o mano para indicar la hora y carecían de una segunda que indicara los cuartos o medias - los minutos sueltos sólo harían su aparición varios siglos más tarde y en contextos de estudios científicos. Otra importante consecuencia de la tecnología horaria en la época que se transluce en La Celestina es la dificultad de saber la hora exacta a menos que se haya oído y contado puntualmente las campanadas que el reloj público daba sólo una vez cada hora. A falta de relojes privados en las casas, no había otra forma de saber la hora que no fuera esas campanadas horarias. El contarlas aparece como una importante habilidad que requiere cierta atención por parte de los personajes. Por ejemplo, antes de su última cita, Calisto pregunta a sus criados:

Calisto: Mozos, ¿qué hora da el reloj? Sempronio: Las diez [...] Calisto: [...] ¿Cómo, desatinado, sabiendo cuánto me va en ser diez o once, me respondías a tiento lo que más aína se te vino a la boca? ¡O cuitado de mí! ¡Si por caso me hobiera dormido y colgara mi pregunta de la respuesta de Sempronio para hacerme de once diez, y así de doce once! (239-40)

Pasajes como éste en que se menciona y se cuentan las campanadas muestran cómo saber la hora es una tarea colectiva, no sólo en el sentido de la necesidad que todos los habitantes tienen de estar coordinados por ella sino también de averiguarla y comunicarla. Esto hace que el reloj mecánico sea un cuasi-personaje en La Celestina, un personaje nunca visto, sólo oído, que guarda silencio la mayor parte del tiempo pero al que es necesario estar siempre atento. Esta situación en la obra muestra la importancia creciente de la medición del tiempo en el mundo citadino sirve de telón de fondo a la obra. Rojas está mostrando la vida de ciudades como Toledo o Salamanca que ya se regían por este tipo de horarios dictados por el reloj. Desde el punto de vista histórico y antropológico, éste es un momento muy especial en el desarrollo de la conciencia moderna del tiempo, es decir, de la obsesión que todavía está con nosotros de saber exactamente qué hora es para poder aprovechar el tiempo al máximo.

11.- Como Lida de Malkiel señaló, esta precisión horaria en la literatura es algo sin precedentes, y sólo las comedias humanísticas Poliodorus y La Veneciana presentan referencias horarias comparables (cit., p. 185). Es curioso que referencias a horas concretas no aparezcan apenas en el primer acto de La Celestina atribuido al Antiguo Autor. Puedes ser simplemente un efecto del desarrollo de la acción o una diferente sensibilidad temporal de los diferentes autores. 
El saber la hora requería un acto de atención continua, un estar al tanto de no perder las campanadas que ha dado el reloj y que no volverán a ocurrir hasta dentro de otra hora completa. Las frecuentes alusiones a las horas sirven así para presentar a los personajes en un estado de continua tensión producto del apremio por estar en todo momento al tanto de la hora en mundo citadino ya altamente coordinado y obsesionado con la economía de una hora exacta que, sin embargo, no es fácil de averiguar. Los personajes de La Celestina están así afectados por una angustia horaria, por la necesidad de estar siempre al tanto de la hora precisa para no encontrarse perdidos en medio del rápido fluir de la jornada urbana. Frente a esta nueva forma de vivir el tiempo, la obra presenta claras alusiones a formas anteriores que aún siguen vigentes. La advertencia de Sosia a Tristán sobre la necesidad de marchar en silencio cuando acompañan a su amo en su nocturna excursión amorosa coloca juntos a diferentes grupos sociales que representan formas marcadamente diferentes de vivir el tiempo: "Tristán, debemos ir muy callando, porque suelen levantarse a esta hora los ricos, los codiciosos de temporales bienes, los devotos de templos, monasterios y iglesias, los enamorados como nuestro amo, los trabajadores de los campos e labranzas [...]» (276). Por las calles aún a oscuras de una ciudad en que casi todos duermen se nos presenta aquí a los mercaderes que madrugan para ir a su negocio y que representan el nuevo y triunfante tiempo mercantil. Junto a ellos van los ciudadanos piadosos que se dirigen a rezar laúdes o maitines a iglesias y conventos antes del alba y que representan el tiempo sagrado y ritual de ascendencia medieval. Igualmente caminan ya por las oscuras calles los campesinos que se han mudado a la ciudad pero que aún se rigen por el tradicional sistema de la hora solar y quieren empezar a trabajar sus tierras con los primeros rayos del sol. Finalmente, están los locos enamorados, que representan una economía hedónica del tiempo regida por el reloj sin horas de conseguir el placer momentáneo del amor. Las concepciones sagradas y hedónicas del tiempo habían ya aparecido enfrentadas concisamente en una imagen de Celestina al describir los efectos que su entrada en la iglesia solía causar entre los eclesiásticos que le habían confiado el cuidado de su mancebas: "De media legua que me viesen dejaban las horas: uno a uno y dos a dos venían adonde yo estaba, a ver si mandaba algo, a preguntarme cada uno por la suya» (215). En este pasaje "las horas» que los eclesiásticos dejan por la consecución de horas de placer terrenal son las horas canónicas, que representan el tiempo sagrado y ritual que está empezando a dejar de ser significativo en los nuevos centros urbanos.

En el plano literario y artístico, el uso colectivo de un horario preciso sirve para que la compleja trama de La Celestina se desarrolle de manera eficaz sin ralentizar el diálogo innecesariamente. La precisión del reloj permite que Celestina establezca en pocas palabras la cita inicial entre Calisto y Melibea («Melibea: [...] Di a qué hora. Celestina: A las doce» 
[229]). Igualmente permite a Calisto y Melibea convenir con rapidez sus citas en noches sucesivas («MelibeA: [...] sea tu venida por este secreto lugar a la mesma hora» [276]). Otros eventos de la obra, como el convite en casa de Celestina, se fijan con la misma rapidez y precisión mediante la eficiencia que permite el reloj. Es más, si no fuera por la existencia del reloj, algunos episodios no podrían tener la notable brevedad que contribuye a su efecto. Éste es el caso con la mortal celada que Centurio tiende a Calisto haciendo que Traso y sus rufianes pasen voceando mientras Calisto está en el huerto y sus criados montan guardia afuera. El encadenamiento de eventos que rápidamente llevan a la muerte de Calisto al bajar por la escala es sólo posible porque el imprudente Sosia había revelado a Areúsa que las citas no eran, como muchos creían, «antes de las diez», cuando se limitaba a llevar a los caballos a beber, sino "en dando el reloj las doce» $(304,305)$.

Además de agilizar la trama, las abundantes referencias al tiempo y la hora precisa cumplen el importante papel de reforzar temas fundamentales de la obra al nivel concreto de las acciones cotidianas y anecdóticas. El tema de la fugacidad del tiempo y de la necesidad de aprovecharlo, $\tan$ representado en proverbios o citas eruditas en boca de los personajes, se ve reforzado por esta presencia de las horas. Es como si las horas que da reloj de la ciudad recordasen regularmente la fugacidad de un tiempo que, además de su valor tradicional de oportunidad terrenal para ganar la salvación eterna, está ahora cargado de utilidad práctica y valor comercial. Igualmente, la necesidad de una economía del tiempo que la división en horas enfatiza está claramente ligada al tema de la fortuna, especialmente en su variante de saber aprovechar la ocasión. Además de las muchas referencias directas a la fortuna, en La Celestina abundan menciones a la necesidad de hacer las cosas a su tiempo debido aprovechando el momento propicio para cada acción. ${ }^{12}$ Así, a las once de la noche, Pármeno sugiere a un enfadado Calisto que no riña sino que, mientras espera la cita con Melibea a las doce, «mejor sería, señor, que se gastase esta hora que queda en aderezar armas que en buscar cuestiones» (240). Una idea similar de oportunidad se transmite en las palabras de Calisto al oír el reloj antes de entrar al huerto de Melibea: "Las doce da ya; buena hora es [...] a buen tiempo llegamos» (241). Pero Celestina es con mucho la mejor economista del tiempo y la oportunidad. Su experiencia le ha enseñado que, a pesar de su regularidad mecánica, el tiempo es una percepción subjetiva y no un valor absoluto. Por ello sabe dilatar las esperas y acelerar las acciones a su conveniencia. Igualmente sabe que el tiempo, como experiencia humana que es, no se presta a cálculos simplistas. Así

12.- La oportunidad u ocasión, especialmente la necesidad de saber tomarla cuando se presenta, está relacionado con el tema de la caprichosa fortuna desde antiguo. Sobre la importancia de la fortuna en la obra, véase, por ejemplo, Erna Ruth Berndt, Amor, muerte y fortuna en "La Celestina", Madrid, Gredos, 1963. 
lo expresa cuando es interpelada por Sempronio, que la espera tras su visita a Melibea: «Sempronio: [...] Que desde que dio la una te espero aquí, y no he sentido mejor señal que tu tardanza. Celestina: Hijo, esa regla de bobos no es siempre cierta, que otra hora me pudiera más tardar y dejar allá las narices, y otras dos [horas], y narices e lengua. Y así que, mientra más tardase, más caro me costase» (139).

Esta sabiduría de Celestina sobre lo relativo del tiempo contrasta con la ignorancia de Calisto, quien, al comunicarle Sosia que Sempronio y Pármeno han sido ejecutados por matar a Celestina, responde incrédulo: «No ha cuatro horas que de mi se despidieron» (267). Y es que la mayoría de los personajes de la obra se caracterizan precisamente por su mal manejo del tiempo y funcionan así como contra-ejemplos en el tema de su aprovechamiento. Con repetida asiduidad los personajes llegan tarde a sus citas, a veces con retrasos de horas cuyo número se indica con precisión en los diálogos. Así ocurre con el retraso con que llegan Pármeno y Sempronio al convite en casa de Celestina y del que Elicia se queja: "[Que] ha tres horas que está aquí mi prima» (203). A una mala administración del tiempo pertenecen también los frecuentes usos en la obra de pasajes que implican que algo se realiza a destiempo. Celestina reza "a donosa hora», según se queja Pármeno que quiere verla (201); igualmente, Celestina visita a Areúsa tan entrada la noche que es calificada por ésta "como huestantigua a tal hora» (173); Pármeno y Calisto llaman a la casa de Celestina de madrugada a pedir su parte y, a sus palabras identificándose como sus hijos, responde: "no tengo yo hijos que anden a tal hora» (254), etc. En algunos casos, el elemento de la oportunidad y la fortuna se entrelazan claramente, como cuando Celestina, ante la ira de Melibea, se dice para sus adentros: «En hora mala acá vine si me falta mi conjuro» (127).

Dada la incompetencia en la economía del tiempo de la mayoría de los personajes no es de extrañar la abundancia del adverbio «tarde» en la obra - dieciocho casos-, a los que hay que añadir varias ocurrencias de "temprano", "madrugar», "presto» y otras expresiones similares usadas en el sentido negativo de demasiado pronto o en momento inadecuado: Alisa confiesa que "es tarde para ir visitar a mi hermana» (117), la cual se encuentra gravemente enferma, quizá ya muerta; tarde llega Pármeno a casa de su amo tras la noche de amor con Areúsa; tarde se levanta Calisto; "tan poco tiempo poseído el placer, tan presto venido el dolor» son palabras de Melibea tras la muerte de Calisto (325); " [t] emprano cobraste los sentimientos de la vejez» le dice Pleberio a una Melibea que se lamenta por la muerte de Calisto antes de subir a la torre (328); «madrugaron a morir» Pármeno y Sempronio en palabras de Sosia (266). La muerte misma de Calisto al caer desde el muro se debe a haberse apresurado: ataviado sólo con su capa y sin haberse puesto la coraza, se apura tanto al descender la escala para ayudar a un Sosia y Tristán que ya no necesi- 
tan de su presencia («Tente, señor, no bajes que idos son» [323]) que se precipita en una caída fatal.

\section{Bibliografía citada}

Arce de Otálora, Juan de. Coloquios de Palatino y Pinciano. Ed. José Luis Ocasar Ariza, 2 vols. Madrid: Turner, 1995.

Asensio, Manuel J. "El tiempo en La Celestina». Hispanic Review 20.1 (1952), pp. 28-43.

Berndt, Erna Ruth. Amor, muerte y fortuna en "La Celestina». Madrid: Gredos, 1963

Cipolla, Carlo M. Clocks and Culture, 1300-1700. London: Collins, 1967.

DoHrn-van Rossum, History of the Hour: Clocks and Modern Temporal Orders. Chicago: University of Chicago Press, 1996.

García-Diego, José A. Los relojes y autómatas de Juanelo Turriano. Madrid: Albatros, 1982.

Gilman, Stephen. "A Propos of 'El tiempo en La Celestina' by Manuel J. Asensio». Hispanic Review 21.1 (1953), pp. 42-45.

Gockerell, Nina. "Tellling Time without a Clock». The Clockwork Universe: German Clocks and Automata 1550-1560. Ed. Klaus Maurice y Otto Mayr. New York: Neal Watson Academic Publishing, 1980, pp. 131-43.

Le Goff, Jacques. "Merchant's Time and Church's Time in the Middle Ages». Time, Work and Culture in the Middle Ages. Ed. Jacques Le Goff. Trad. Arthur Goldhammer. Chicago: University of Chicago Press, 1980, pp. 29-42.

Lida de Malkiel, María Rosa. La originalidad artística de "La Celestina». Buenos Aires: Eudeba, 1962.

Maravall, José Antonio. El mundo social de "La Celestina». Madrid: Gredos, 1964.

Montañés Fontenla, Luis, ed. Biblioteca literaria del relojero. 6 vols. Madrid: R. Carbonell Blasco, 1953-1958.

RojAS, Fernando de, y "Antiguo Autor». La Celestina, tragicomedia de Calisto y Melibea. Ed. Guillermo Serés, Francisco J. Lobera, Paloma DíazMas, Carlos Mota, Iñigo Ruiz Arzálluz y Francisco Rico. Barcelona: Crítica, 2000.

Sevilla Arroyo, Florencio. «Amor, magia y tiempo en La Celestina». Celestinesca 33 (2009), pp. 173-214.

Von Bertele, H. «Precision Timekeeping in the Pre-Huygens Era». The Horological Journal 95 (1953), pp. 794-816. 


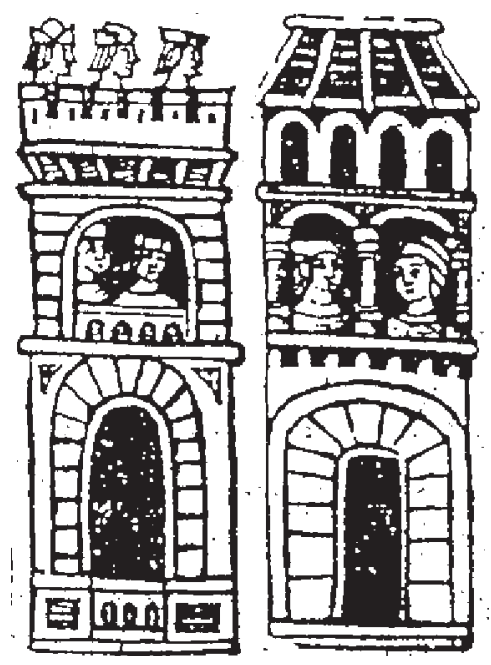




\section{FERNÁNDEZ Rivera, Enrique, «El reloj, la hora y la economía del tiempo en La Celestina», Celestinesca, 34 (2010), pp. 31-40.}

\section{RESUMEN}

La Celestina refleja fidedignamente una época en la que, al no abundar relojes de uso privado, un reloj público regía con sus campanadas la ya apresurada vida citadina. Esto sirve de telón de fondo a la acción, la agiliza y contribuye a varios de sus temas centrales. La compulsión por saber la hora y la dificultad en averiguarla de los personajes, el continuo desfase entre su percepción del paso del tiempo y el ritmo inalterable del reloj mecánico son parte del conocido malestar existencial de $\mathrm{La} \mathrm{Ce}$ lestina. Las campanadas de este reloj nunca visto pero siempre presente refuerzan los temas tan importantes en la obra de la fugacidad del tiempo y la necesidad de aprovechar la ocasión cuando se presenta.

PALABRAS ClaVE: Celestina, tiempo, reloj, horario, hora.

\section{ABSTRACT}

Celestina faithfully portraits an era when the bells of a public clock - private clocks were rare- ruled the by then fast-paced urban life. The clock and its marking of time provide the backdrop to the action, make the development of the plot agile, and reinforce several central themes. The characters' compulsion to know the time and their difficulties in determining it, as well as the disagreement between their perception of the time passing and the inflexible rhythm of the mechanical clock, contribute to the famous existential malaise in Celestina. The clock, which is never seen but is always present, reinforces also the central themes of the fugacity of time, and the importance of grabbing the opportunity when it knocks.

KEY WORDS: Celestina, time, clock, telling time, hour. 